\title{
Correction to: Management of duodenal stump fistula after gastrectomy for malignant disease: a systematic review of the literature
}

\author{
Maurizio Zizzo ${ }^{1,2^{*}}$, Lara Ugoletti ${ }^{3}$, Lorenzo Manzini ${ }^{1}$, Carolina Castro Ruiz ${ }^{3}$, Gabriela Elisa Nita ${ }^{1}$, Magda Zanelli ${ }^{4}$, \\ Loredana De Marco ${ }^{4}$, Giulia Besutti ${ }^{5}$, Rocco Scalzone ${ }^{1}$, Romano Sassatelli ${ }^{6}$, Valerio Annessi ${ }^{3}$, Antonio Manenti ${ }^{7}$ and \\ Claudio Pedrazzoli ${ }^{1}$
}

\section{Correction to: BMC Surg (2019) 19:55 https://doi.org/10.1186/s12893-019-0520-x}

Following publication of the original article [1], the authors have notified us that due to administrative reasons they would like to modify the first affiliation from:

- AUSL-IRCCS di Reggio Emilia

To:

- Azienda Unità Sanitaria Locale - IRCCS di Reggio Emilia

Author details

'Department of Oncology and Advanced Technologies, Surgical Oncology Unit, Arcispedale Santa Maria Nuova di Reggio Emilia, Azienda Unità Sanitaria Locale - IRCCS di Reggio Emilia, 42123 Reggio Emilia, Italy. ${ }^{2}$ Clinical and Experimental Medicine PhD Program, University of Modena and Reggio Emilia, Modena, Italy. ${ }^{3}$ General and Emergency Surgery Unit, Ospedale Civile di Guastalla, Azienda Unità Sanitaria Locale - IRCCS di Reggio Emilia, 42123 Reggio Emilia, Italy. ${ }^{4}$ Department of Oncology and Advanced Technologies, Pathology Unit, Arcispedale Santa Maria Nuova di Reggio Emilia, Azienda Unità Sanitaria Locale - IRCCS di Reggio Emilia, 42123 Reggio Emilia, Italy. ${ }^{5}$ Department of Imaging and Laboratory Medicine, Radiology Unit, Arcispedale Santa Maria Nuova di Reggio Emilia, Azienda Unità Sanitaria Locale - IRCCS di Reggio Emilia, 42123 Reggio Emilia, Italy. ${ }^{6}$ Department of Oncology and Advanced Technologies, Gastrointestinal Endoscopy Unit, Arcispedale Santa Maria Nuova di Reggio Emilia, Azienda Unità Sanitaria Locale - IRCCS di Reggio Emilia, 42123 Reggio Emilia, Italy. ${ }^{7}$ Department of General Surgery, Azienda Ospedaliero-Universitaria Policlinico, Del Pozzo Street 71, 41124 Modena, Italy.

\footnotetext{
* Correspondence: zizzomaurizio@gmail.com

'Department of Oncology and Advanced Technologies, Surgical Oncology Unit, Arcispedale Santa Maria Nuova di Reggio Emilia, Azienda Unità Sanitaria Locale - IRCCS di Reggio Emilia, 42123 Reggio Emilia, Italy

${ }^{2}$ Clinical and Experimental Medicine PhD Program, University of Modena and Reggio Emilia, Modena, Italy

Full list of author information is available at the end of the article
}

Published online: 24 October 2019

\section{Reference}

1. Zizzo, et al. Management of duodenal stump fistula after gastrectomy for malignant disease: a systematic review of the literature. 2019, 2019;19:55. https://doi.org/10.1186/s12893-019-0520-x.

(c) The Author(s). 2019 Open Access This article is distributed under the terms of the Creative Commons Attribution 4.0 International License (http://creativecommons.org/licenses/by/4.0/), which permits unrestricted use, distribution, and reproduction in any medium, provided you give appropriate credit to the original author(s) and the source, provide a link to the Creative Commons license, and indicate if changes were made. The Creative Commons Public Domain Dedication waiver (http://creativecommons.org/publicdomain/zero/1.0/) applies to the data made available in this article, unless otherwise stated. 